\title{
Put more trust in the trustworthy and less in the untrustworthy to improve judgement of medicines
}

An editorial error changed the meaning in this letter "Put more trust in the trustworthy and less in the untrustworthy to improve judgement of medicines" by Mary Madden (BMJ 2017;358:j4202, doi:10.1136/bmj.j4202).

The penultimate sentence should have read, "Pervasive use of a 'deficit model,' which implies that all public and professional scepticism of science is unfounded and that corrective communication by experts is necessary, is unhelpful" [not "Pervasive discussion of the 'deficit model,' which implies that all public and professional scepticism of science is unfounded and that corrective communication by experts is necessary, is unhelpful," as published]. 